informed the development of modern physical treatments for psychiatric disorders, in particular depression (Malhi et al. Neuropsychiatric Dis Treatment 2006, 2 165-179). We describe a study of direct current stimulation for the clinical treatment of depression and its neurobiological effects as measured electrophysiologically, in a pilot study conducted at the Black Dog Institute. Transcranial direct current stimulation (tDCS) is a noninvasive technique in which a weak direct current is applied across the scalp to alter the excitability of juxtaposed cortical tissue. The effects on neuronal membranes and neurotransmission persist beyond the periods of stimulation and can be measured using quantitative EEG. Preliminary findings from seven subjects will be presented and the putative mechanism of action discussed. In addition, the literature pertaining to this field will be reviewed with reference to current research in tDCS and emergent findings from modern deep brain stimulation and neurosurgical interventions (Dalgleish et al. Am J Psychiatry 2004, 161 1913-1916).

\section{Different Approaches to Endophenotypes in Schizophrenia}

\section{E Scarr}

Centre for Neuroscience, The University of Melbourne, Victoria, Australia

\section{Overview}

The concept that schizophrenia is a grouping of, rather than a single illness, is well accepted. However, it is seldom explored and rarely taken into account when investigating the disorder. This symposium is structured to give a snapshot of different concepts of endophenotypes, starting with those arising from basic research, progressing to an endophenotype associated with a purported risk factor for schizophrenia. We then move into the clinical setting, addressing whether or not testing paradigms define discrete groupings of altered functionality. The symposium ends with a presentation on endophenotypes defined by cognitive testing and the genetic aspect of such deficits.

08-01

\section{Neurochemical endophenotypes of schizophrenia}

\section{E Scarr ${ }^{1,2}$}

${ }^{1}$ Centre for Neuroscience, The University of Melbourne, Victoria, Australia; and ${ }^{2}$ The Rebecca L. Cooper Research Laboratories, The Mental Health Research Institute, Victoria, Australia
A major problem for investigators in the field of schizophrenia research is the difficulty of producing unambiguous results because, at least in part, of schizophrenia being a syndrome comprising of a number of disorders, which all present clinically with similar clusters of symptoms. The symptoms of schizophrenia can be categorized into three clusters: 1) positive symptoms (an excess or distortion of normal functions), 2) negative symptoms (the diminution or loss of normal functions) and 3) cognitive symptoms (deficits in attention, concentration and memory) (American Psychiatric Association, Diagnostic and Statistical Manual of Mental Disorders 2000). The heterogeneity of the schizophrenia syndrome ordains that studies on the disorder generate data that have a 'decreased signal to noise ratio' (Hallmayer et al. Am J Hum Genet 2005,77 468-476). That is to say, studying the biochemical indexes of a group of disorders gives a less clear outcome than would be obtained by studying a single disorder. It has now been shown that investigating specific phenotypes in the schizophrenia syndrome and comparing results across phenotypes within the syndrome, as well as to those from control subjects, enhances the potential of identifying specific pathogenetic mechanisms (Hallmayer et al. Am J Hum Genet 2005, 77 468-476). We now use this approach of using endophenotypes to increase the capacity of our postmortem research to detect the biological abnormalities that underlie the schizophrenia syndrome.

08-02

\section{Identifying Disease-specific Protein Expression Patterns Within the Syndrome of Schizophrenia}

\section{B Dean ${ }^{1,2,3}$}

'The Mental Health Research Institute; ${ }^{2}$ The University of Melbourne Departments of Psychiatry and Pathology, Parkville; and ${ }^{3}$ The Department of Psychological Medicine, Monash University, Clayton, Victoria, Australia

While schizophrenia has long been recognized as a syndrome, no strong biological basis for segregating the diseases within that syndrome has been elucidated. One of the defining outcomes of disease is changes in the biochemical pathways affected by the disorder. Such changes would be predicted to alter levels of critical proteins in these disease-specific pathway changes. Two-dimensional (2D) electrophoresis now provides the opportunity to identify changes in the levels of multiple proteins in complex biological symptoms and therefore offers the opportunity to identify disease-specific protein footprints in tissue affected by different diseases. This approach has 
already been used to identify proteins that are affected in tumors and hence lead to specific targets for drugs to treat different forms of cancer. In a similar approach, 2D electrophoresis is being carried out using postmortem central nervous system (CNS) from a large cohort of subjects with schizophrenia and tissue from the same CNS region of subjects with no history of psychiatric or neurological disease. We have modified the approach to 2D electrophoresis so that the analyses will measure the levels of over 10000 proteins in each tissue sample. In collaboration with the Centre for Mathematics and Statistics of Complex Systems, we are now analyzing our 2D gels to identify grouping of differential protein expression patterns that might define different illnesses within the syndrome of schizophrenia. In this presentation, a proof of principal study to differentiating schizophrenia and bipolar disorder will be presented.

$08-03$

\section{From biological marker to endophenotype: the role of animal models}

\author{
D Eyles ${ }^{1,2}$, T Burne' ${ }^{2}$ J McGrath ${ }^{2,3}$ \\ 'School of Biomedical Sciences, University of Queensland; ${ }^{2}$ Queensland Centre for \\ Mental Health Research, The Park, Wacol, Queensland, Australia; and \\ ${ }^{3}$ Department of Psychiatry, University of Queensland, Brisbane, Australia
}

Our group has been exploring the concept that developmental vitamin D (DVD) deficiency may be the plausible neurobiological explanation for several important epidemiological correlates of schizophrenia, namely 1) the excess winter/spring birth rate, 2) increased incidence of the disease in second-generation Afro-Caribbean migrants and 3) increased urban birth rate. We have produced two pieces of direct support for this hypothesis in patients. DVD deficiency therefore is a plausible 'biological marker' for schizophrenia. We have recently resolved a major technological barrier that will now allow us to test this hypothesis in a major European developmental biobank. The genetic factors or heritability indicators that would endow DVD deficiency as a valid schizophrenia endophenotype are emerging. Our group has established a highly informative animal model to study the effects of DVD deficiency on brain development. This animal model reproduces the gross pathological features of the disease, that is, ventriculomegaly as well as sensitivity to amphetamine- and MK-801-induced hyperlocomotion and impairments in latent inhibition, behaviours analogous to the positive and negative symptoms of the disease in patients. We are now examining whether this model internally fulfils certain molecular endophenotypic criteria. Our initial data are encouraging as we find a $36 \pm 11 \%$ decrease in the developmental expression of the candidate endophenotypic gene for schizophrenia, COMT. This animal model continues to provide good face and some construct validity with the disease in patients and illustrates how animal models can be used to progress plausible biological markers for schizophrenia into endophenotypes.

08-04

\section{Endophenotypic biobehavioural markers for schizophrenia: how close are we to finding the Holy Grail? \\ P Ward ${ }^{1,2}$ \\ 'University of New South Wales, Sydney, New South Wales, Australia; and ${ }^{2}$ Schizophrenia Research Unit, Sydney South West Area Health Service, Liverpool, Australia}

Research over several decades has showed that current operational diagnoses are unlikely to map directly onto the biological substrates of schizophrenia. This has led to increased interest in identifying endophenotypic markers that may provide an intermediate link to underlying genes. This talk will review some of the more promising biobehavioural markers currently being investigated, such as P50 sensory gating, reduced mismatch negativity amplitude and regionally specific gray matter volume loss, and highlight some of the challenges that still remain to be overcome. These include the potential impact of various potential confounds (eg treatment effects and comorbidity). Our current state of knowledge highlights the need for large-scale collaborative efforts that could provide convincing evidence for the utility of putative biobehavioural markers in furthering our understanding of the etiology of schizophrenia.

\section{8-05}

\section{'Kraepelinian' and 'Bleulerian' schizophrenia: a genetic dissection of a cognitive endophenotype}

\section{A Jablensky $y^{1,2}, \mathrm{~J}$ Hallmayer ${ }^{1,3}, \mathrm{M}$ Dragovic ${ }^{2}$, J Badcock ${ }^{1,2}$, L Kalaydjieva ${ }^{4}$}

${ }^{1}$ UWA School of Psychiatry and Clinical Neuroscience; ${ }^{2}$ Centre for Clinical Research in Neuropsychiatry; ${ }^{3}$ Stanford University School of Medicine Department of Psychiatry and Behavioural Sciences; and ${ }^{4}$ Western Australian Institute of Medical Research, Perth, Australia 